\title{
Myocardial Injury After Temporary Transvenous Cardiac Pacing
}

\author{
Meng Liu (iD ${ }^{1,2}$ \\ Pingsheng $\mathrm{Wu} \mathbb{D}^{2}$ \\ 'Emergency Department, Hunan \\ Provincial People's Hospital, Medical \\ School of Hunan Normal University, \\ Changsha, People's Republic of China; \\ ${ }^{2}$ Cardiology Department, Nanfang \\ Hospital, Southern Medical University, \\ Guangzhou, People's Republic of China
}

Correspondence: Pingsheng Wu Cardiology Department, Nanfang Hospital, Southern Medical University, No. 1838, North Guangzhou Road, Guangzhou, People's Republic of China Tel +8602061641502

Email wupingshengnf@sina.com
Introduction: Temporary transvenous cardiac pacing (TTCP) can lead to potential penetration and occasional perforation of the right ventricular wall. No study to date has analyzed the effect of TTCP on myocardial injury by cardiac troponin (cTn) measurement. The present study was designed to investigate perioperative myocardial injury in elective TTCP in noncardiac surgical settings.

Patients and Methods: This retrospective study investigated the data collected from August 2018 through March 2020 from 22 eligible patients who underwent elective TTCP for noncardiac procedures. The patients had a median age of $66(50-83)$ years; six $(27.3 \%)$ of them were women, and all of them had a baseline cTn $<1$ upper reference limit (URL). Cardiac biomarker assays were performed before and after TTCP, and their results were compared.

Results: After TTCP, cTn > 1 URL was detected in $20(91 \%, \mathrm{~N}=22)$ patients. Among these 22 patients, paired $t$-test comparing assay results before and after TTCP lead insertion showed a mean cTn elevation of 3.599 URL (95\% CI: 1.566 to 5.632, $\mathrm{P}<0.01$ ), and a mean creatine kinase-MB isoform elevation of 0.1550 URL (95\% CI: -0.01239 to $0.3224, \mathrm{P}>0.05)$.

Conclusion: The study demonstrates a high incidence of myocardial injury associated with TTCP, which should be a matter of concern for the involved physicians.

Keywords: temporary pacemaker, temporary transvenous cardiac pacing, myocardial injury, bradycardia, perioperative myocardial injury

\section{Introduction}

Temporary transvenous cardiac pacing (TTCP) is a potentially life-saving intervention and can be broadly categorized as emergency or elective according to its indications. Emergency TTCP is indicated primarily in life-threatening bradycardia with hemodynamic compromise. Elective TTCP is usually undertaken in surgical settings or for other interventions if the patient's underlying pathology may lead to transient bradycardia or the subsequent procedure is likely to produce bradycardia. ${ }^{1,2}$ However, TTCP has limited coverage in consensus guidelines, and most available evidence has been derived from small observational studies and clinical experience rather than scientific trials. ${ }^{2,3}$

Complications of TTCP can be classified into two broad categories: venous access-related complications or pacing catheter-related complications. ${ }^{4}$ Ultrasoundguided venous access has been shown to be safe; thus, complications associated with this method are rare. ${ }^{5-7}$ It is well known that the implantation of a permanent pacemaker is associated with minor myocardial injury, which is typically quantified 
by measuring serum cardiac troponin (cTn) levels. ${ }^{8-10}$ The lead fixation mechanism during permanent pacemaker implantation may inevitably damage the myocardium, ${ }^{8,10}$ while a standard TTCP pacing catheter has a blunt, smooth tip and lacks a fixation mechanism. Is the implantation of a standard TTCP pacing catheter also associated with myocardial injury? The standard TTCP pacing lead is relatively stiff, and placement of a pacing lead within the right ventricle may promote ventricular ectopic activity and occasionally prolong ventricular arrhythmia. ${ }^{11}$ This pacing lead can penetrate and occasionally perforate the right ventricular wall; ${ }^{2,12}$ rarely, this will result in cardiac tamponade associated with an increased risk for inhospital death. ${ }^{13}$ Perforated and penetrated ventricular walls are signs of substantial myocardial injury, a term used when there is evidence of elevated cTn values at least one value above the 99th percentile upper reference limit (URL) in the current guidelines. ${ }^{14}$ However, there is no study evaluating myocardial injury associated with TTCP by cTn assays. Patients requiring emergency TTCP usually have an acute heart condition such as myocardial infarction, heart failure, cardiogenic shock, or cardiac arrest, ${ }^{13}$ which subsequently cause $\mathrm{cTn}$ to increase. Postoperative cTn levels are elevated after all types of cardiac surgeries; ${ }^{15}$ therefore, to exclude other factors affecting cTn release as much as possible, this study was designed to investigate perioperative myocardial injury in elective TTCP in the setting of noncardiac surgeries.

\section{Patients and Methods Study Design and Patients}

This retrospective study involved 33 consecutive patients who underwent elective TTCP for scheduled noncardiac surgeries performed by the first author from August 2018 to March 2020. The cardiovascular risk for each patient was low, except for those with bradycardia; thus, the TTCP lead was placed at the bedside by the first author, who is an emergency physician and only experienced in TTCP lead placement at bedside; in other situations not encountered in this study, cardiologists have to operate on the patient for lead placement in a catheter lab. Among the 33 enrolled patients, 22 had cardiac biomarkers measured both before and after TTCP placement and compared.

The twenty-two eligible patients had a median age of 66 years (range: 50-83 years), and six (27\%) were female. Thirteen (59\%) surgical patients underwent tumor excision, including two with colon cancer, two with rectal cancer, three with lung cancer, three with maxillofacial cancer, two with stomach cancer, and one with bladder cancer. Among the other nine surgical cases, one had an inguinal hernia, three had a cervical fracture or cervical spinal disease, one had intestinal obstruction, and four had a biliary stone. None of the patients had a history of myocardial infarction, stroke, heart failure, or chronic kidney disease. No remarkable structural abnormalities were shown on preoperative transthoracic echocardiography in any of the patients. Except for slightly elevated creatinine in one patient $(120.5 \mu \mathrm{mol} / \mathrm{L})$, B-type natriuretic peptide (BNP), hemoglobin and creatinine levels were normal. The decision to perform TTCP was made by the responsible doctors after consultation with cardiologists and/or anesthesiologists. TTCP indications and baseline patient characteristics are included in Table 1.

Table I Baseline Characteristics of Patients and Indication of TTCP

\begin{tabular}{|c|c|}
\hline Characteristics & All $(\mathbf{N}=\mathbf{2 2})$ \\
\hline Age, y [range (median)] & $55-83(66)$ \\
\hline Female, n (\%) & $6(27.3)$ \\
\hline Hypertension, n (\%) & $3(13.6)$ \\
\hline Diabetes mellitus, n (\%) & $3(13.6)$ \\
\hline Coronary artery disease, $\mathrm{n}(\%)$ & I (4.5) \\
\hline Smoking, n (\%) & $8(36.4)$ \\
\hline Surgery type, n (\%) & \\
\hline Tumor resection & $13(59.1)$ \\
\hline Bile duct stones & $4(18.2)$ \\
\hline Spine diseases & $3(13.6)$ \\
\hline Intestinal obstruction & I (4.5) \\
\hline Hernia & I (4.5) \\
\hline Indication, n (\%) & \\
\hline Mobitz type II AVB & I (4.5) \\
\hline Sinus pause & I (4.5) \\
\hline Sinus bradycardia complicated with: & \\
\hline Junctional rhythm & I (4.5) \\
\hline SVT/AT & $2(9.1)$ \\
\hline $1^{\circ} \mathrm{AVB} \& \mathrm{CRBBB}$ & I (4.5) \\
\hline Potential intraoperative bradycardia & $12(54.5)$ \\
\hline Negative atropine challenge test & $4(18.2)$ \\
\hline
\end{tabular}

Notes: Sinus bradycardia: sinus rate $<50 \mathrm{bpm}$. Potential intraoperative bradycardia: certain surgical procedures potentially cause periods of bradycardia by activating the trigeminal cardiac reflex or vagus nerve, including maxillofacial surgeries (one patient), peritoneal insufflation/perfusion chemotherapy (five patients), and procedures that involve manipulation of the spine (two patients) or bile duct (four patients).

Abbreviations: AVB, atrioventricular block; CRBBB, complete right bundle branch block; SVT, supraventricular tachycardia; AT, atrial tachycardia. 
TTCP catheters were placed within 12 hours before surgery for all 22 patients, maintained for 6 to 24 hours, and withdrawn soon after surgery for 21 patients; the final patient was maintained on TTCP for 3 days. Cardiac biomarker values were measured within two weeks before TTCP and at night (two patients) or the next morning (20 patients) after the surgery. Additional cTn assays were performed post TTCP for three patients.

\section{Material and Temporary Pacemaker Placement}

Temporary pacemakers (model 5348 or 5392, Medtronic), 7 French (Fr) hemostasis introducers (Fast-Cath ${ }^{\mathrm{TM}}$ \& Cath-Lock ${ }^{\mathrm{TM}}$, St. Jude Medical), and 6 Fr nonfloating (without a balloon) right heart curve bipolar pacing catheters (PACEL $^{\mathrm{TM}}$, St. Jude Medical) were used.

The most common access site was the right internal jugular vein (16 patients, 73\%) followed by the subclavian vein (four patients, $18 \%$ ) when the surgery involved the right neck. The femoral vein approach was used (two patients, 9\%) after difficulties were experienced in advancing the TTCP catheter through the subclavian vein or right internal jugular vein. Except for subclavian vein access, all central venous access was performed under ultrasound guidance.

Pacing catheterization was guided by intracavity electrocardiography (IC-ECG). ${ }^{16,17}$ The target of the proximal electrode was shown by a slight ST-segment elevation $<2 \mathrm{mV}$ on IC-ECG, constituting a proper positioning against the ventricular wall and an adequate pacing site, ${ }^{18}$ which can be very difficult to maintain it if the patient changes positions (Figure 1). The location of the catheter tip was further confirmed with downward QRS waves in leads II, III, and aVF on standard pacing ECG.
The sensing threshold ranged from $2.5 \mathrm{mV}$ to $20 \mathrm{mV}$ (maximum setting at the pacemaker), with an average of $7.34 \mathrm{mV}$. The pacing threshold was $2 \mathrm{~mA}$ in one patient and no more than $1 \mathrm{~mA}$ for the others, with an average of $0.72 \mathrm{~mA}$. The pacemaker was set to VVI mode, at a rate of 40 to $60 \mathrm{ppm}$, a sense of 1.5 to $3 \mathrm{mV}$, and an average output of 3.0 (from 2 to 8 ) $\mathrm{mA}$.

Few complications were observed clinically. One patient felt uncomfortable when the test pacing was at 80 pulses per minute. Arterial puncture, which did not require particular intervention, occurred accidentally for two patients, including one with a hematoma of the thorax caused by subclavian artery puncture. Ventricular ectopic beat and ventricular tachycardia were common when the pacing catheter was moved in the right ventricle chamber to achieve a proper ST-elevation myocardial infarction (STEMI) pattern (ST-segment elevation $<2 \mathrm{mV}$ ) on ICECG. These were resolved once manipulation of the lead ceased. Other complications, such as thromboembolism, infection, and bleeding, were not observed.

\section{Statistical Analysis}

Data on the plasma concentrations of cardiac biomarkers, including high-sensitivity $\mathrm{cTn}$, creatine kinase-MB isoform (CK-MB), creatine kinase (CK), and myoglobin (MYO), were analyzed. The cTn assay $(\mathrm{N}=44)$ results included cTnT (normal range $<14 \mathrm{pg} / \mathrm{mL}$ ) for nine assays and cTnI (normal range $<0.03 \mathrm{ng} / \mathrm{mL}$ ) for 35 assays. The measurement units were standardized to the URL. The number of patients with myocardial injury and CK-MB above the URL was determined. The paired $t$-test was applied to compare the four biomarker values before and after TTCP. For all analyses, $\mathrm{P}<0.05$ indicated statistical significance. The analyses were conducted using GraphPad Prism 5.

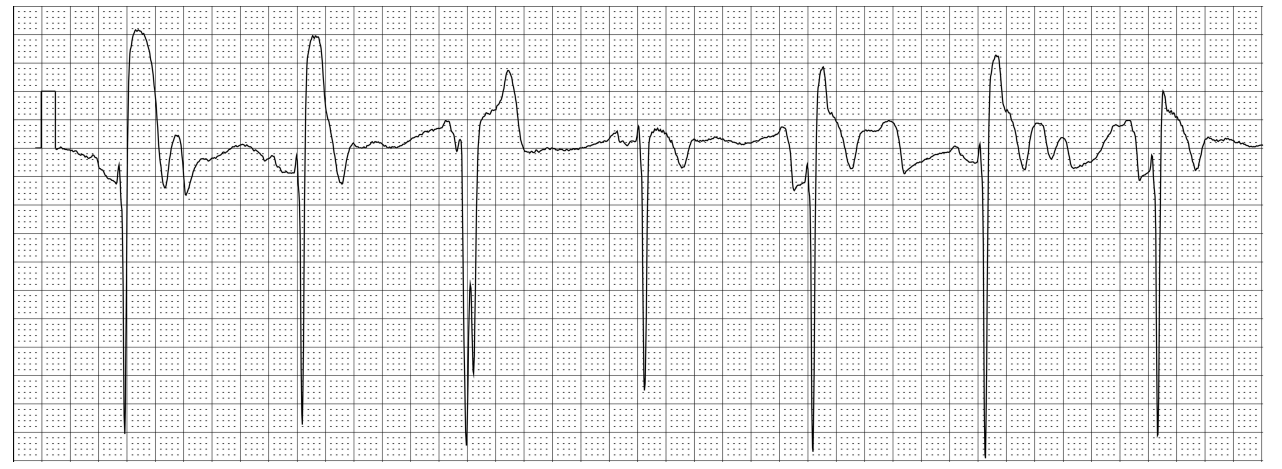

Figure I Access site through the right jugular vein. On IC-ECG, the ST-segment elevation varies when the patient turns his or her head from right to left. 


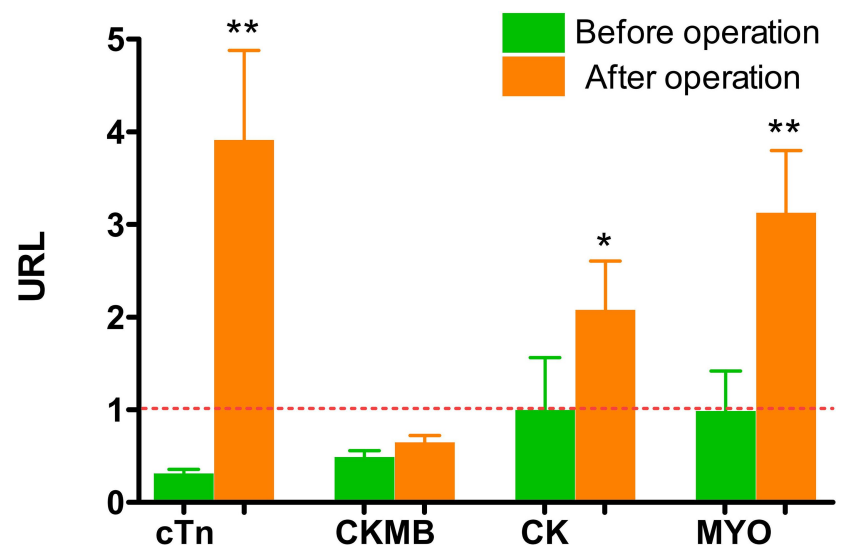

Figure 2 Comparisons of cTn, CK-MB, CK, and MYO levels before and after the operation $* \mathrm{P}>0.05, * * \mathrm{P}<0.01$.

Abbreviation: URL, upper reference limit.

\section{Ethical Consideration}

This study followed the guidelines established in the Helsinki Declaration. The Hunan Provincial People's Hospital Ethics Committee granted study approval for the study (reference number: 2020-07) and waived the usual requirement for informed consent, as all data were deidentified and analyzed anonymously.

\section{Results}

Myocardial injury was identified in 20 (91\%) of the 22 eligible patients: the cTn level before TTCP was below the URL, ie, cTn< 1 URL; after TTCP, $20(91 \%, N=22)$ patients demonstrated $\mathrm{cTn}>1 \mathrm{URL}$.

Among the 22 patients, there were statistically significant differences in the serum cTn and MYO levels before and after the operation: cTn mean of differences 3.599 URL (95\% CI: 1.566 to 5.632), $\mathrm{P}=0.0014$; MYO mean of differences 2.139 URL (95\% CI: 0.8034 to 3.475 ), $\mathrm{P}=0.0032$. There were no statistically significant differences in the serum CK-MB and CK levels before and after the operation: CK-MB mean of differences 0.1550 URL ( $95 \%$ CI: -0.01239 to 0.3224 ), $\mathrm{P}=0.0677$; $\mathrm{CK}$ mean of differences $1.080 \mathrm{URL}$ (95\% CI: -0.4072 to 2.568 ), $\mathrm{P}=0.1458$ (Figure 2).

Three patients received repeated cTn assays after TTCP. Specifically, the cTn level returned to normal on the third day and the fifth day after TTCP for one patient each, while for the final patient, the cTn level was slightly above 1 URL on the third day after TTCP, but no further assays were performed. Four patients underwent postoperative ECG recording, none of which demonstrated ischemic changes.

\section{Discussion}

In this study, perioperative myocardial injury was detected by an increase in high-sensitivity cTn in 20 of 22 (91\%) patients. Before the cTn assay and the term myocardial injury were introduced, the best myocardial infarction biomarker was CK-MB. In this study, CK-MB was slightly above 1 URL for only four $(18 \%)$ of the 22 patients, and the average increase of $0.1550 \mathrm{URL}$ was not statistically or clinically significant, which may partly explain why a high incidence of myocardial injury after TTCP has not been previously reported. The elevated CK and MYO values could be attributed to perioperative noncardiac muscle injury.

Risks of myocardial injury, including hypoxemia, shock/hypotension, anemia, renal failure, heart failure, and ischemic heart disease, ${ }^{14}$ were not recorded for the 22 patients. In patients with high cardiovascular risk, perioperative myocardial injury detected by an acute increase in high-sensitivity cTn is a common complication after noncardiac surgery, occurring in $13 \%$ to $16 \%$ of patients; ${ }^{19,20}$ subsequent subgroup analysis of those patients with active tumor disease identified perioperative myocardial injury in $12 \%$ to $19 \% .{ }^{19,20}$ In our study, in patients with similar or even less cardiovascular risk, perioperative myocardial injury was identified in $91 \%$ of patients, which raises concerns of myocardial injury by TTCP.

Is the pacing current the cause of the elevated cTn level? The average pacing threshold and current of the TTCP in this study were as small as those of permanent pacemakers. Several studies on permanent pacemakerassociated myocardial injury suggest that the mechanical effects of the pacing lead rather than pacing current result in an elevation of cTn. ${ }^{8-10}$ Christopher and others reported that after permanent cardiac pacing, a small rise in cTn levels above normal was observed in $21 \%$ of patients, which was only correlated with a prolonged time for lead implantation. ${ }^{8}$ The much higher incidence of myocardial injury in our study indicates that standard temporary leads cause greater trauma to the myocardium than permanent pacing leads. Unlike these leads, which cause injury to the myocardium only at the time of implantation, standard temporary leads may frequently cause friction and injury to a specific area of the cardiac endocardium.

In this study, most indications for TTCP could be identified in patients who were at high risk for the development of intraoperative or periprocedural bradycardia because of their 
characteristics or procedure type. These are traditional, clinical experience-based, rather than evidence-based, indications, ${ }^{2}$ and there is no clear consensus or specific recommendation for TTCP in the current guidelines. ${ }^{1}$ The benefits of TTCP include eliminating bradycardia and preventing cardiac arrest. TTCP lead placement is generally safe and has a low pericardial complication rate. ${ }^{13}$ Because of the safety of well-prepared elective TTCP performed by an experienced operator, in this study's hospital, elective TTCP is performed prophylactically for patients at high risk for the development of bradycardia undergoing surgery as a practical measure. Emergency TTCP, however, may be poorly prepared or performed by an inexperienced operator. For example, only $7 \%$ of emergency TTCP operators had ever received any formal training in the $\mathrm{UK}^{21}$ In our study, even though TTCP was well prepared and IC-ECG guidance was used to ensure that the pacing lead properly touched the right ventricle wall, ${ }^{16-18}$ a high incidence of myocardial injury was still demonstrated.

In emergency TTCP, the myocardium may be more vulnerable to the pacing catheter. As Hwang and others report, in acute inferior STEMI and high-degree atrioventricular block, periprocedural TTCP for primary percutaneous coronary intervention does not decrease the incidence of adverse cardiocerebrovascular events and thus is more likely to increase the incidence of cardiogenic shock and prolong hospitalization. $^{22}$ An analysis of $>360,000$ patients who underwent TTCP in the United States between 2004 and 2014 concluded that TTCP is generally safe with low pericardial complication rates $(0.6 \%){ }^{13}$ However, the inhospital mortality rate $(>10 \%)$ and strikingly high mortality rate $(>50 \%)$ over 4 years of follow-up are concerning and suggest the need to identify other complications and alterations of TTCP. ${ }^{4,13,23}$ Myocardial injury is prognostically important and warrants clinical attention. ${ }^{24-26}$ This may not necessarily apply to myocardial injury associated with TTCP, whose underlying pathophysiological mechanisms may differ from those of other causes of myocardial injury, such as myocardial infarction, shock, or myocarditis. Nevertheless, TTCP placement should be prudent regarding myocardial injury, which complies with the guideline recommendation that TTCP should be avoided or applied as briefly as possible. ${ }^{1,27}$ Whether standard temporary leads cause massive cardiac endothelial damage is currently unknown and is worthy of further study in animal models.

Theoretically, from the hardware perspective, a more flexible pacing lead with a softer tip might help mitigate or avoid myocardial injury. In this study, the temporary catheters had a smooth, $6 \mathrm{Fr}$, isodiametric profile with no fixation mechanism (standard catheters), enabling sufficient stiffness to prevent dislocation and easy removal. Lang et al reported that TTCP with floating catheters (more flexible than standard catheters) were associated with lower complication rates, ${ }^{28,29}$ but according to a survey on current practices, only approximately $16 \%$ of the interviewed physicians preferred floating catheters. ${ }^{30}$ The use of an externalized permanent active fixation lead in place of the standard temporary pacing lead in TTCP has been suggested to have a safer profile $^{31,32}$ and is recommended by the current guidelines for patients who require prolonged TTCP. ${ }^{1}$ A new TTCP lead with a soft distal tip to minimize the risk of cardiac injury and retractable wire loop stabilizers that provide atraumatic myocardial fixation were demonstrated to be safe and technically feasible. ${ }^{33}$

This study has several limitations. First, the patient cohort was small and not part of a consecutive series, and temporary pacing was performed by an emergency physician, resulting in insufficient power for generalizing the results to a wider population. Second, mixed cTnI and cTnT results were analyzed by standardizing the units to URL, which is in accord with the criteria of the definition of myocardial injury in the current guidelines; therefore, although the qualitative diagnosis of myocardial injury for all patients was appropriate, the quantitative analysis was not accurate. Third, our study did not determine whether or how a rise in cTn level is correlated with adverse clinical events. Last, the pacing leads were not monitored by echocardiography, which can be used to observe the real-time movement of the pacing wire or possible lead friction on the right ventricle endocardium. A combination of cardiac biomarker assays and echocardiography monitoring should reveal more information in future studies of TTCP.

\section{Conclusion}

Although our study has several limitations, the results indicate that the TTCP catheter should be considered more than a foreign body. Due to the high incidence of myocardial injury, clinicians are advised to consider it in decision-making for TTCP implantation.

\section{Abbreviations}

URL, upper reference limit; TTCP, temporary transvenous cardiac pacing; cTn, cardiac troponin; IC-ECG, intracavity electrocardiography; CK-MB, creatine kinase $\mathrm{MB}$ isoform; CK, creatine kinase; MYO, myoglobin. 


\section{Funding}

This research did not receive any specific grant from funding agencies in the public, commercial, or not-forprofit sectors.

\section{Disclosure}

The authors declare no conflicts of interest or financial involvement with this manuscript.

\section{References}

1. Kusumoto FM, Schoenfeld MH, Barrett C, et al. 2018 ACC/AHA/ HRS guideline on the evaluation and management of patients with bradycardia and cardiac conduction delay: a report of the American College of Cardiology/American Heart Association task force on clinical practice guidelines and the heart rhythm society. Circulation. 2019;140(8):e382-e482. doi:10.1161/ CIR.0000000000000628

2. Gammage MD. Temporary cardiac pacing. Heart. 2000;83 (6):715-720. doi:10.1136/heart.83.6.715

3. Fitzpatrick A, Sutton R. A guide to temporary pacing. BMJ. 1992;304 (6823):365-369. doi:10.1136/bmj.304.6823.365

4. Tjong FVY, de Ruijter UW, Beurskens NEG, Knops RE. A comprehensive scoping review on transvenous temporary pacing therapy. Neth Heart J. 2019;27(10):462-473. doi:10.1007/s12471019-01307-x

5. Gangathimmaiah V. Emergency transvenous cardiac pacing. Emerg Med Australas. 2017;29(2):229-232. doi:10.1111/1742-6723.12757

6. Piela N, Kornweiss S, Sacchetti A, Gallagher A, Abrams A. Outcomes of emergency department placement of transvenous pacemakers. Am J Emerg Med. 2016;34(8):1411-1414. doi:10.1016/ j.ajem.2016.04.017

7. Birkhahn RH, Gaeta TJ, Tloczkowski J, et al. Emergency medicine-trained physicians are proficient in the insertion of transvenous pacemakers. Ann Emerg Med. 2004;43(4):469-474. doi:10.1016/j.annemergmed.2003.09.019

8. Boos CJ, Gough S, Wheather M, Medbak S, More R. Effects of transvenous pacing on cardiac troponin release. Pacing Clin Electrophysiol. 2004;27(9):1264-1268. doi:10.1111/j.15408159.2004.00618.x

9. Nikolaou NI, Christou AH, Spanodimos SG, Antonatos DG, Korkonikitas PI, Patsilinakos SP. Marked troponin elevation after implantation of a permanent antibradycardia pacemaker. Hellenic J Cardiol. 2011;52(6):489-492.

10. Blazek P, Ferri-Certic J, Vrazic H, et al. Pacemaker implantation associated myocardial micro-damage: a randomised comparison between active and passive fixation leads. Sci Rep. 2018;8(1):4870. doi:10.1038/s41598-018-23209-5

11. Austin JL, Preis LK, Crampton RS, Beller GA, Martin RP. Analysis of pacemaker malfunction and complications of temporary pacing in the coronary care unit. Am J Cardiol. 1982;49(2):301-306. doi:10.1016/0002-9149(82)90505-7

12. Hynes JK, Holmes DR Jr., Harrison CE. Five-year experience with temporary pacemaker therapy in the coronary care unit. Mayo Clin Proc. 1983;58(2):122-126.

13. Metkus TS, Schulman SP, Marine JE, Eid SM. Complications and outcomes of temporary transvenous pacing: an analysis of $>360,000$ patients from the national inpatient sample. Chest. 2019;155 (4):749-757. doi:10.1016/j.chest.2018.11.026

14. Thygesen K, Alpert JS, Jaffe AS, et al. Fourth universal definition of myocardial infarction (2018). Eur Heart J. 2019;40(3):237-269.
15. Horvath KA, Parker MA, Frederiksen JW, Palmer AS, Fullerton DA. Postoperative troponin I values: insult or injury? Clin Cardiol. 2000;23(10):731-733. doi:10.1002/clc.4960231009

16. Liu M, Han X. Bedside temporary transvenous cardiac pacemaker placement. Am J Emerg Med. 2019;38(4):819-822. doi:10.1016/j. ajem.2019.12.013

17. Bing OH, McDowell JW, Hantman J, Messer JV. Pacemaker placement by electrocardiographic monitoring. $N$ Engl J Med. 1972;287 (13):651. doi:10.1056/NEJM197209282871309

18. Goldberger J, Kruse J, Ehlert FA, Kadish A. Temporary transvenous pacemaker placement: what criteria constitute an adequate pacing site? Am Heart J. 1993;126(2):488-493. doi:10.1016/0002-8703(93) 91083-Q

19. Puelacher C, Lurati Buse G, Seeberger D, et al. Perioperative myocardial injury after noncardiac surgery: incidence, mortality, and characterization. Circulation. 2018;137(12):1221-1232. doi:10.1161/ CIRCULATIONAHA.117.030114

20. Costa M, Furtado MV, Borges FK, et al. Perioperative troponin screening identifies patients at higher risk for major cardiovascular events in noncardiac surgery. Curr Probl Cardiol. 2019;46 (3):100429. doi:10.1016/j.cpcardiol.2019.05.002

21. Sharma S, Sandler B, Cristopoulos C, Saraf S, Markides V, Gorog DA. Temporary transvenous pacing: endangered skill. Emerg Med J. 2012;29(11):926-927. doi:10.1136/emermed-2011-200044

22. Hwang YM, Kim CM, Moon KW. Periprocedural temporary pacing in primary percutaneous coronary intervention for patients with acute inferior myocardial infarction. Clin Interv Aging. 2016;11:287-292. doi:10.2147/CIA.S99698

23. Ng ACC, Lau JK, Chow V, Adikari D, Brieger D, Kritharides L. Outcomes of 4838 patients requiring temporary transvenous cardiac pacing: a Statewide Cohort Study. Int J Cardiol. 2018;271:98-104. doi:10.1016/j.ijcard.2018.05.112

24. Morrow DA. The fourth universal definition of myocardial infarction and the emerging importance of myocardial injury. Circulation. 2020;141(3):172-175. doi:10.1161/CIRCULATIONAHA.119.0 44125

25. Sarkisian L, Saaby L, Poulsen TS, et al. Clinical characteristics and outcomes of patients with myocardial infarction, myocardial injury, and nonelevated troponins. Am J Med. 2016;129(4):446e445-446 e421. doi:10.1016/j.amjmed.2015.11.006

26. Sarkisian L, Saaby L, Poulsen TS, et al. Prognostic impact of myocardial injury related to various cardiac and noncardiac conditions. $\mathrm{Am}$ J Med. 2016;129(5):506-514e501. doi:10.1016/j.amjmed.2015.12.009

27. Brignole M, Auricchio A, Baron-Esquivias G, et al. 2013 ESC guidelines on cardiac pacing and cardiac resynchronization therapy: the task force on cardiac pacing and resynchronization therapy of the European Society of Cardiology (ESC). Developed in collaboration with the European Heart Rhythm Association (EHRA). Eur Heart J. 2013;34(29):2281-2329. doi:10.1093/eurheartj/eht150

28. Ferguson JD, Banning AP, Bashir Y. Randomised trial of temporary cardiac pacing with semirigid and balloon-flotation electrode catheters. Lancet. 1997;349(9069):1883. doi:10.1016/S01406736(97)24026-2

29. Lang R, David D, Klein HO, et al. The use of the balloon-tipped floating catheter in temporary transvenous cardiac pacing. Pacing Clin Electrophysiol. 1981;4(5):491-496. doi:10.1111/j.15408159.1981.tb06216.x

30. Diemberger I, Massaro G, Rossillo A, et al. Temporary transvenous cardiac pacing: a survey on current practice. $J$ Cardiovasc Med (Hagerstown). 2020;21(6):420-427. doi:10.2459/JCM.000000000 0000959

31. Braun MU, Rauwolf T, Bock M, et al. Percutaneous lead implantation connected to an external device in stimulation-dependent patients with systemic infection-a prospective and controlled study. Pacing Clin Electrophysiol. 2006;29(8):875-879. doi:10.1111/j.15408159.2006.00454.x 
32. Chihrin SM, Mohammed U, Yee R, et al. Utility and cost effectiveness of temporary pacing using active fixation leads and an externally placed reusable permanent pacemaker. Am J Cardiol. 2006;98 (12):1613-1615. doi:10.1016/j.amjcard.2006.07.041
33. Webster M, Pasupati S, Lever N, Stiles M. Safety and feasibility of a novel active fixation temporary pacing lead. $J$ Invasive Cardiol. 2018;30(5):163-167.

\section{Publish your work in this journal}

Therapeutics and Clinical Risk Management is an international, peerreviewed journal of clinical therapeutics and risk management, focusing on concise rapid reporting of clinical studies in all therapeutic areas outcomes, safety, and programs for the effective, safe, and sustained use of medicines. This journal is indexed on PubMed Central, CAS,
EMBase, Scopus and the Elsevier Bibliographic databases. The manuscript management system is completely online and includes a very quick and fair peer-review system, which is all easy to use. Visit http://www.dovepress.com/testimonials.php to read real quotes from published authors. 